\title{
Monoclonal Immunoglobulin-Associated Renal Lesions in Patients with Newly Diagnosed Multiple Myeloma: A Report from a Single Center
}

\author{
Zi-Shan Lin (D) ${ }^{1, *}$ \\ Xiao-Juan $\mathrm{Yu}^{\mathrm{l}, *}$ \\ Xu Zhang iD ${ }^{1,2}$ \\ Su-Xia Wang ${ }^{1,2}$ \\ Xi-Nan Cen ${ }^{3}$ \\ Fu-De Zhou' \\ Ming-Hui Zhao ${ }^{1,4}$ \\ 'Renal Division, Department of Medicine, \\ Peking University First Hospital; Institute of \\ Nephrology, Peking University, Renal \\ Pathology Center, Institute of Nephrology, \\ Peking University, Key Laboratory of Renal \\ Disease, Ministry of Health of China, Key \\ Laboratory of CKD Prevention and \\ Treatment, Ministry of Education of China, \\ Beijing, People's Republic of China; \\ ${ }^{2}$ Laboratory of Electron Microscopy, \\ Pathological Centre, Peking University First \\ Hospital, Beijing, People's Republic of \\ China; ${ }^{3}$ Department of Hematology, Peking \\ University First Hospital, Beijing, People's \\ Republic of China; ${ }^{4}$ Peking-Tsinghua \\ Center for Life Sciences, Beijing, People's \\ Republic of China
}

*These authors contributed equally to this work

Correspondence: Fu-De Zhou Renal Division, Department of Medicine, Peking University First Hospital, Institute of Nephrology, Peking University; Renal Pathology Center, Institute of Nephrology, Peking University, Key Laboratory of Renal Disease, Ministry of Health of China, Key Laboratory of CKD Prevention and Treatment, Ministry of Education of China, No. 8 Xishiku Street, Xicheng District, Beijing, 100034, People's Republic of China $\mathrm{Tel}+86-1350 \mid$ I 62527

Fax +86-10-83575694

Email zhoufudel801@vip.sina.com
Background: Monoclonal immunoglobulin-associated renal lesions in patients with newly diagnosed myeloma vary. We aimed to determine the pathological spectrum and analyze associated prognostic factors.

Methods: Fifty-six patients with newly diagnosed multiple myeloma and biopsy-proven renal lesions were enrolled. Kidney biopsies were reanalyzed, and the baseline clinical characteristics, treatments and outcomes were recorded.

Results: Fifty-one patients had monoclonal immunoglobulin-associated renal lesions, with myeloma cast nephropathy (MCN) being the most common pattern. We divided our cohort into pure $\mathrm{MCN}, \mathrm{MCN}+$ other pathologies and non-MCN. Patients with $\mathrm{MCN}$ had more severe renal injury than those with non-MCN. In our cohort, none of the patients with pure $\mathrm{MCN}$ or MCN + other pathologies presented with nephrotic syndrome. Patients with nonMCN had better renal and overall survival than those with pure MCN but similar survivals to those with $\mathrm{MCN}+$ other pathologies. Number of myeloma casts (HR 1.08, p = 0.012) was the only independent prognostic factor for renal survival. Male sex (HR: 3.64; $p=0.015)$ and number of casts (HR: 1.17; $\mathrm{p}=0.001$ ) were independent prognostic factors for overall survival.

Conclusion: Patients with MCN had more severe renal injury than those with non-MCN. Patients with non-MCN had better renal and overall outcomes than those with pure MCN, but their outcomes were similar to those with $\mathrm{MCN}+$ other pathologies. The independent predictors of overall survival were male sex and number of myeloma casts.

Keywords: kidney biopsy, multiple myeloma, myeloma cast nephropathy, monoclonal immunoglobulin, prognosis

\section{Introduction}

Multiple myeloma (MM) is a hematological malignancy derived from the abnormal proliferation of clonal plasma cells, often resulting in organ dysfunction. ${ }^{1}$ Depending on the definition used, the incidence of renal involvement in MM, which is associated with poor outcomes, ranges from $20 \%$ to $50 \%{ }^{2,3}$

Renal failure in most patients with $\mathrm{MM}$ is caused by the nephrotoxicity of monoclonal immunoglobulin. ${ }^{3}$ The spectrum of renal lesions caused directly by monoclonal immunoglobulin in patients with $\mathrm{MM}$ includes myeloma cast nephropathy (MCN), monoclonal immunoglobulin deposition disease (MIDD), and amyloidosis. ${ }^{4,5}$ Other rarer patterns of renal lesions have also been reported. ${ }^{6}$ Patients with different patterns of renal lesions have different manifestations, ${ }^{7-10}$ 
and those with pure MCN present with more severe renal injury. ${ }^{7,9,10}$ The International Myeloma Working Group (IMWG) suggests that in patients with high serum free light chain levels and predominant light chain proteinuria, the most likely diagnosis is $\mathrm{MCN}$ and kidney biopsy is unnecessary. ${ }^{11}$ However, increasing evidence shows that kidney biopsy has significance for prognostic evaluation because the extent of cast formation, interstitial fibrosis and tubular atrophy (IFTA) are associated with renal survival in patients with MCN. ${ }^{12,13}$

Previous studies have shown that patients with kidney biopsy proven $\mathrm{MCN}$ or MCN combined with light chain deposition disease (LCDD) had worse outcomes than those with pure LCDD, ${ }^{9,10}$ but patients with monoclonal gammopathy of renal significance (MGRS) in those studies were not excluded. The manifestation and outcome of pure LCDD may be influenced by the absence of MM.

Here, we reviewed and analyzed 56 Chinese patients with newly diagnosed MM and renal involvement who had undergone kidney biopsy at Peking University First Hospital from 1999 to 2017. Our study aimed to determine the pathological spectrum, clinical characteristics and prognostic factors of monoclonal immunoglobulin-associated renal lesions in newly diagnosed myeloma patients.

\section{Materials and Methods}

\section{Patients}

The medical records of newly diagnosed MM patients between 1999 and 2017 were reviewed. An ultrasoundguided percutaneous native kidney biopsy was performed in 56 patients. Indications for kidney biopsy were acute kidney injury or acute kidney disease (64\%), new-onset proteinuria or proteinuria accompanied by hematuria (22\%), and nephrotic syndrome (14\%). With the result of kidney biopsy, we recognized the underlying disease maybe MM or other hematological malignancy. Then we perform bone marrow smear and biopsy to confirm. The diagnosis of MM was strictly established depending on the guidelines of the IMWG. ${ }^{14}$

The study was approved by the Ethics Committee of our hospital and complied with the Declaration of Helsinki. Each patient provided informed consent.

\section{Clinical Data}

Demographic, clinical, laboratory, treatment and outcome data were collected. The following clinical manifestations were defined: hypoalbuminemia (serum albumin $<30 \mathrm{~g} / \mathrm{L}$ ), nephrotic-range proteinuria (proteinuria $>3.5 \mathrm{~g} / 24 \mathrm{~h}$ ), nephrotic syndrome (serum albumin $<30 \mathrm{~g} / \mathrm{L}$ with proteinuria $>3.5 \mathrm{~g} / 24 \mathrm{~h}$ ), hypercalcemia (serum calcium $>2.5 \mathrm{mmol} / \mathrm{L}$ ), acute kidney disease (changes in renal structure or function for less than 3 months), acute kidney injury (a $\geq 50 \%$ increase in the serum creatinine level occurring over 1-7 days or the presence of oliguria for more than 6 hours), anuria (urine output $<100 \mathrm{~mL} / 24 \mathrm{~h}$ ), oliguria (urine output $100-400 \mathrm{~mL} / 24 \mathrm{~h}$ ), and worsening renal function (a decrease of more than $30 \mathrm{~mL} / \mathrm{min} / 1.73 \mathrm{~m}^{2}$ at admission in eGFR). The endpoints included end-stage renal disease (ESRD) and death.

\section{Renal Histopathology}

All biopsies were available for review. Kidney biopsy specimens were stained and analyzed by light microscopy, immunofluorescence and electron microscopy. Two pathologists evaluated the renal biopsy specimens separately.

According to a previous study, for patients with MCN, the mean number of myeloma casts per $\times 200$ microscopic fields was recorded. ${ }^{12}$ The scores of tubular atrophy, interstitial fibrosis, interstitial inflammation and acute tubular injury were assessed as follows: $<10 \%(0), 10-24 \%$ (1), $25-50 \%$ (2), $>50 \%$ (3). ${ }^{13}$ The presence of interstitial edema was also assessed. ${ }^{13}$

\section{Statistical Analysis}

All statistical analyses were performed using SPSS version 24.0 (SPSS, Armonk, NY, USA). $\mathrm{P}<0.05$ was considered statistically significant. For normally distributed data, the mean \pm standard deviation was used to describe the quantitative variables and $t$ test was used to compare the differences in quantitative parameters. For non-normally distributed data, the median with the range was used to describe the quantitative variables, and the Mann-Whitney $U$-test was used to compare the differences in quantitative parameters. Categorical data were described by absolute percentages, and differences between 2 groups were compared using $\chi 2$ test. The Kaplan-Meier method was used to plot the survival curves, which were statistically compared using the Log rank test. The associations between variables and outcomes were analyzed using a Cox regression model. The hazard ratios (HRs) with 95\% confidence intervals (CIs) and $p$ values were reported. 


\section{Results}

\section{Patient Characteristics}

Three hundred ninety-seven MM patients with a mean age of $60.3 \pm 11.4$ years were newly diagnosed at our center (234 men and 163 women) between 1999 and 2017. Two hundred fifty-six patients $(64 \%)$ had renal involvement (serum creatinine $>178 \mu \mathrm{mol} / \mathrm{L}$ or proteinuria). Kidney biopsy was only performed in $56 / 256$ patients $(22 \%)$. The baseline clinical characteristics of these patients are summarized in Table 1. The patients who had undergone kidney biopsy were younger and had significantly higher hemoglobin levels than those without kidney biopsy.

\section{Pathological Characteristics of Monoclonal Immunoglobulin-Associated Renal Lesions}

Fifty-one patients had monoclonal immunoglobulinassociated lesions and five patients had non-monoclonal immunoglobulin-associated lesions. Eighteen patients with monoclonal immunoglobulin-associated lesions (35\%) were $\kappa$-restricted (Table 2). MCN was the most common monoclonal immunoglobulin-associated renal lesion in 31 patients, including 2 with the coexistence of amyloidosis (Figure 1) and 4 with the coexistence of LCDD. Two patients with pure MCN exhibited diffuse amyloid casts, which were $\lambda$-restricted. Other renal histopathologic diagnoses included pure amyloidosis (9 patients), pure LCDD (5 patients), light and heavy chain deposition disease ( 2 patients), light chain proximal tubulopathy ( 2 patients including one patient with coexisting C3 glomerulopathy), and fibrillary glomerulonephritis (2 patients). Non-monoclonal immunoglobulin-associated lesions were observed in 5 patients.

Next, we divided our cohort into three groups: pure $\mathrm{MCN}$ $(n=25), M C N+$ other pathologies $(n=6)$ and non-MCN $(n=20)$. As shown in Table 3, patients with $\mathrm{MCN}+$ other pathologies (median score, 2.5) had more severe acute tubular injury than those with non-MCN (median score, 1), but they showed no significant difference compared with those with pure MCN (median score, 2). Patients with pure MCN had significantly higher scores of tubular atrophy than those with $\mathrm{MCN}+$ other pathologies and those non-MCN $(\mathrm{p}=0.001)$. Patients with non-MCN had significantly lower scores of interstitial fibrosis

Table I Baseline Clinical Characteristics of Patients with Renal Involvement

\begin{tabular}{|c|c|c|c|}
\hline Parameters & Patients without Kidney Biopsy & Patients with Kidney Biopsy & $\mathbf{p}$ \\
\hline Number of patients & 200 & 56 & \\
\hline Male/female & $124 / 76$ & $35 / 21$ & 0.946 \\
\hline Age $(y)$ & $60 \pm 12$ & $55 \pm 11$ & 0.003 \\
\hline Hemoglobin $(g / L)$ & $91.0(40.0,168.0)$ & $94.3(70.0,162.0)$ & 0.03 \\
\hline Serum creatinine $(\mu \mathrm{mol} / \mathrm{L})$ & $182.5(31.0,2361.0)$ & $182.5(31.0,1294.1)$ & 0.729 \\
\hline eGFR $\left(\mathrm{mL} / \mathrm{min} / \mathrm{l} .73 \mathrm{~m}^{2}\right)$ & $30.54(1.69,118.23)$ & $30.35(2.5,114.68)$ & 0.842 \\
\hline Calcium (mmol/L) & $2.3(1.7,3.9)$ & $2.3(1.8,3.5)$ & 0.06 \\
\hline \multicolumn{4}{|l|}{ Heavy chain type } \\
\hline $\operatorname{lgG}(\%)$ & $83(41.5 \%)$ & $13(23.2 \%)$ & 0.012 \\
\hline $\lg A(\%)$ & 37 (18.5\%) & $8(14.3 \%)$ & 0.464 \\
\hline $\lg M(\%)$ & 0 & $\mathrm{I}(\mathrm{I} .8 \%)$ & 0.219 \\
\hline $\lg \mathrm{D}(\%)$ & $2(1 \%)$ & 0 & 1.0 \\
\hline None (\%) & $78(39 \%)$ & $34(60.7 \%)$ & 0.004 \\
\hline \multicolumn{4}{|l|}{ Light chain type } \\
\hline$\kappa(\%)$ & 89 (44.5\%) & 19 (33.9\%) & 0.157 \\
\hline$\lambda(\%)$ & III (55.5\%) & $37(66.1 \%)$ & 0.157 \\
\hline Proteinuria (\%) & $76(38 \%)$ & 22 (39\%) & 0.861 \\
\hline Microscopic hematuria (\%) & $62(31 \%)$ & 16 (29\%) & 0.691 \\
\hline
\end{tabular}


Table 2 Kidney Biopsy Findings of Monoclonal Immunoglobulin-Associated Renal Lesions in 5I NDMM Patients

\begin{tabular}{|c|c|c|}
\hline Pathological Diagnosis & Number of Patients (\%) & Light Chain Isotype $(\kappa / \lambda)$ \\
\hline Pure MCN & $25(49 \%)$ & $7 / 18$ \\
\hline MCN concurrent with amyloidosis & $2(4 \%)$ & $0 / 2$ \\
\hline MCN concurrent with LCDD & $4(8 \%)$ & $3 / 1$ \\
\hline Pure LCDD & $5(10 \%)$ & $3 / 2$ \\
\hline Pure amyloidosis & $9(17 \%)$ & $1 / 8$ \\
\hline Light and heavy chain deposition disease & $2(4 \%)$ & $2(\lg G \kappa) / 0$ \\
\hline LCPT & $\mathrm{I}(2 \%)$ & $\mathrm{I} / 0$ \\
\hline C3 glomerulopathy concurrent with LCPT & I (2\%) & $0 / 1$ \\
\hline Fibrillary glomerulonephritis & $2(4 \%)$ & $1 / 1$ \\
\hline
\end{tabular}

Abbreviations: NDMM, newly diagnosed multiple myeloma; MCN, myeloma cast nephropathy; LCDD, light chain deposition disease; light and heavy chain deposition disease; LCPT, light chain proximal tubulopathy; C3, complement factor 3 .

than those with pure $\mathrm{MCN}$ or $\mathrm{MCN}+$ other pathologies $(\mathrm{p}=$ $0.008)$.

\section{Clinical Characteristics of Patients with Kidney Biopsy Proven Monoclonal Immunoglobulin-Associated Renal \\ Lesions}

The clinical characteristics at the time of kidney biopsy are listed in Table 4. The male/female ratio was $34 / 17$, with a mean age of $55 \pm 11$ years. The median hemoglobin level was 93 (range: 70-162) $\mathrm{g} / \mathrm{L}$, and it was significantly higher in the non-MCN group $(114.5 \mathrm{~g} / \mathrm{L})$ than in the pure $\mathrm{MCN}$ group $(83.0 \mathrm{~g} / \mathrm{L})$, but was not significantly different from that in the $\mathrm{MCN}+$ other pathologies group $(90.5 \mathrm{~g} / \mathrm{L})$. The median serum creatinine level was $272 \mu \mathrm{mol} / \mathrm{L}$ (range: $31.0-1294.1$ ) at the time of kidney biopsy, and it was significantly higher in patients with pure $\mathrm{MCN}(606.9 \mu \mathrm{mol} / \mathrm{L})$ or with $\mathrm{MCN}+$ other pathologies $(325.3 \mu \mathrm{mol} / \mathrm{L})$ than in those with nonMCN $(135.1 \mu \mathrm{mol} / \mathrm{L})$. Patients with pure MCN (56\%) had a higher incidence of dialysis requirements than those with
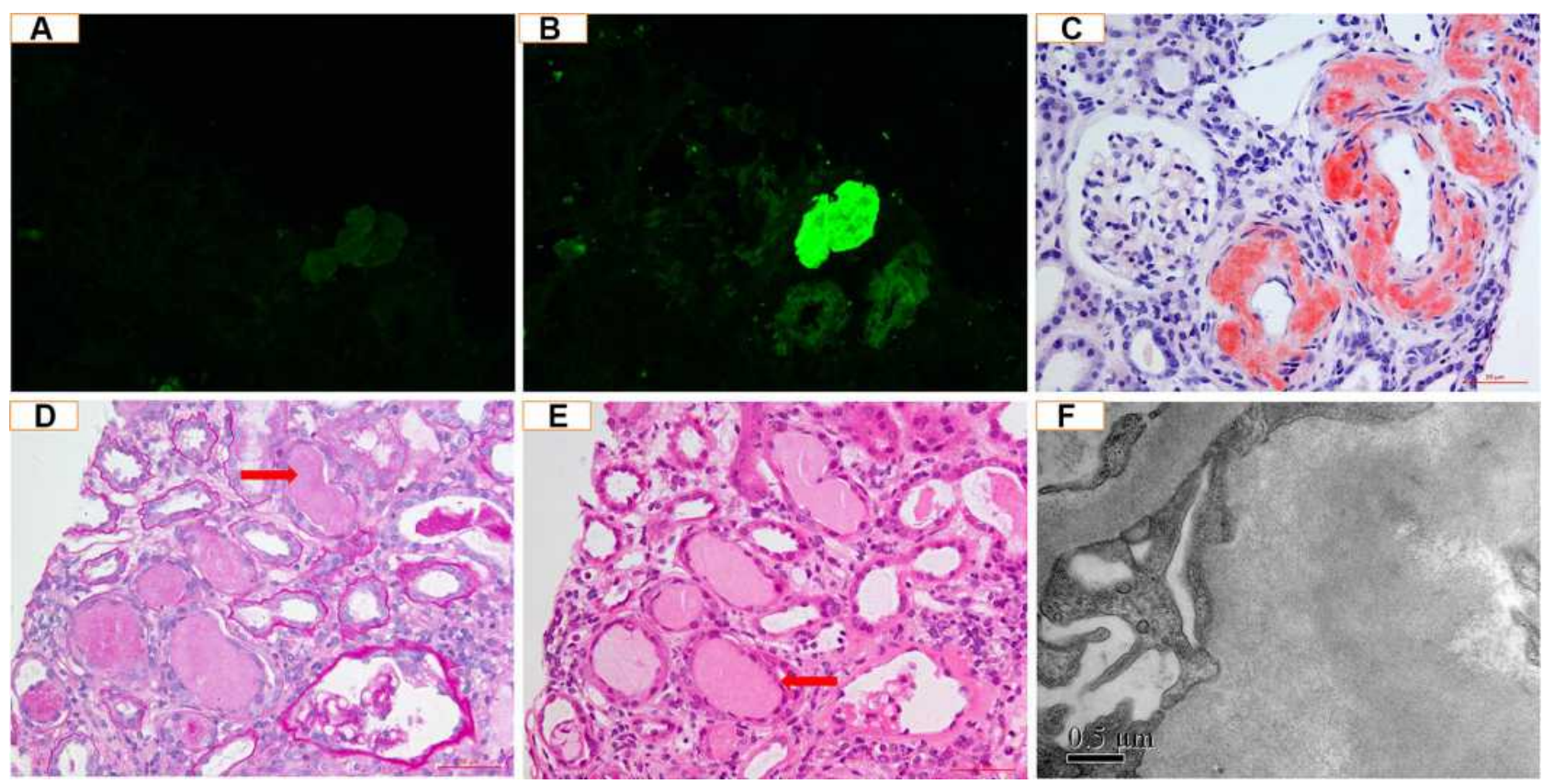

Figure I A patient with light chain cast nephropathy and light chain amyloidosis. $(\mathbf{A})$ showed $\kappa$ light chain was negative on the arteriolar wall and protein cast $(\times 200)$; $(\mathbf{B})$ showed $\lambda$ light chain was positive on the arteriolar wall and strong positive on the protein cast $(\times 200)$; (C) showed Congo-red positive amyloid on the arteriolar/artery wall and weak positive in the glomeruli ( $\times 400)$; (D) showed PAS-negative protein casts in tubular lumen (arrow, $\times 200$, periodic acid-Schiff staining); (E) showed cell infiltration around the protein casts (arrow, $\times 200$, hematoxylin and eosin staining); (F) showed non-branch fibrils in the subepithelial area of glomeruli $(\times 40,000)$. 
Table 3 Pathological Characteristics of Monoclonal Immunoglobulin-Associated Renal Lesions at Diagnosis

\begin{tabular}{|l|l|l|l|}
\hline Parameters & Pure MCN & MCN + Other Pathologies & Non-MCN \\
\hline Number of patients & 25 & 6 & 20 \\
Median number of casts per $\times 200$ fields & $9.5(4.6-3 \mathrm{I})$ & $8.4(4.1-13.8)$ & 0 \\
Acute tubular injury & $2(0-4)$ & $2.5(2-3)$ & $\mathrm{I}(\mathrm{I}-4)$ \\
Tubular atrophy & $\mathrm{I}(0-4)$ & $0(0-1)$ & $0(0-4)$ \\
Interstitial fibrosis & $2(0-4)$ & $2(2,2)$ & $\mathrm{I}(0,3)$ \\
Interstitial inflammation & $2(0-4)$ & $2.5(2-3)$ & $\mathrm{I}(0-3)$ \\
Interstitial edema & $15(60 \%)$ & $1(17 \%)$ & $3(15 \%)$ \\
\hline
\end{tabular}

Abbreviation: $\mathrm{MCN}$, myeloma cast nephropathy.

non-MCN (10\%), but there was no significant difference was found compared with those with $\mathrm{MCN}+$ other pathologies $(30 \%)$.

The median proteinuria level was $3.8 \mathrm{~g} / 24 \mathrm{~h}$ (range: 0-23.5), but no significant difference was found among the three groups. Nephrotic syndrome was observed in 8 patients (16\%). Urine protein electrophoresis was studied in 30 patients, and the extent of albuminuria was higher in those with non-MCN (73.6\%). The incidence of the albumin fraction ( $>50 \%$ total urine protein) was significantly higher in the non-MCN group (92\%), but no significant difference was found in the other albumin fractions $(<10 \%$ and $10-50 \%$ total urine protein). The rate of hematuria was higher in the non-MCN group (48\%) than in the pure MCN (12.5\%) and MCN+ other pathologies $(0 \%)$ groups.

Immunofixation electrophoresis of serum and urine was performed in 50 patients. Forty-six patients (92\%) were positive by serum and/or urine immunofixation electrophoresis. Whole monoclonal immunoglobulin was identified in 17 patients (34\%), and isolated monoclonal free light chains were detected in 33 patients (66\%). No significant difference was found in the M-protein types among the three groups, but patients with MCN were more likely to have isolated serum-free light chains.

All the patients were assessed for extrarenal organ involvement. Eighteen patients exhibited extrarenal manifestations. Thirteen patients had bone lesions ( 7 patients with pure MCN, 3 patients with MCN + other pathologies and 3 patients with non-MCN), but no significant difference was found among the three groups $(\mathrm{p}=0.208)$. Cardiac involvement was identified in 4 patients ( 2 patients with renal amyloidosis, one patient with $\mathrm{MCN}+$ amyloidosis and one patient with LCDD). Liver involvement was identified in one patient with renal amyloidosis. Liver and cardiac involvement was diagnosed in one patient with amyloidosis. Patients with MCN had a significantly higher percentage of plasma cells in diagnostic aspirate smears than those with non-MCN $(\mathrm{p}=0.013)$.

\section{Treatment, Follow-Up and Outcome}

The follow-up data were unavailable in 6 patients. The remaining 45 patients (88\%) were followed up for a median time of 18 months (range: 2-109). All the patients received chemotherapy after kidney biopsy. For patients with MCN or anuria/oliguria, supportive therapy was administrated (urinary alkalization and free access to water). As shown in Table 5, the use of proteasome inhibitors or other chemotherapy was evenly distributed among the three groups. Among 37 patients who were treated with proteasome inhibitors, 6 received high-dose melphalan followed by autologous peripheral blood stem cell transplantation (HDM/ASCT). Eight patients were treated with other chemotherapy regimens, including the VAD regimen (vinorelbine, pirarubicin and dexamethasone) in 5 patients and MPT regimen (melphalan, prednisone and thalidomide) in 3 patients. Among the 15 patients who were on dialysis at diagnosis, 3 patients with $\lambda$ restricted pure-MCN discontinued dialysis for a period of 3, 9 and 12 months. Fifteen patients (33\%) progressed to ESRD within a median duration of one month (range 1-60).

The rate of ESRD in the pure MCN group (50\%) was significantly higher than that in the non-MCN group (11\%), but it was not significantly different from that in the $\mathrm{MCN}+$ other pathologies group (40\%). The median renal survival was 6.9 months in the pure $\mathrm{MCN}$ group and was significantly shorter than that in the non-MCN group (not reached); however, it was not significantly different from that in the $\mathrm{MCN}+$ other pathologies groups (not reached; Figure 2A). Cox regression revealed that the predictors of progression to ESRD in the univariate analysis were the serum creatinine level at diagnosis and 
Table 4 Clinical Characteristics of Patients with Kidney Biopsy-Proven Monoclonal Immunoglobulin-Associated Renal Lesions at Diagnosis

\begin{tabular}{|c|c|c|c|c|}
\hline Parameters & Pure MCN & MCN + Other Pathologies & Non-MCN & $\mathbf{p}$ \\
\hline Number of patients & 25 & 6 & 20 & \\
\hline Male/female & $|4 / 1|$ & $4 / 2$ & $14 / 6$ & 0.664 \\
\hline Age (y) & $52 \pm 10$ & $55 \pm 6$ & $56 \pm 13$ & 0.682 \\
\hline Hemoglobin (g/L) & $83.0(70.0-119.0)$ & $90.5(81.0-133.0)$ & II $4.5(80.0-162.0)$ & $0.006^{\mathrm{a}}$ \\
\hline Serum albumin $(g / L)$ & $42.6(32.2-51.4)$ & $40.0(35.4-46.9)$ & $26.3(15-50.1)$ & $0.002^{\mathrm{a}}$ \\
\hline Hypoalbuminemia & 0 & 0 & II (55\%) & $<0.00 I^{a, b}$ \\
\hline Serum creatinine $(\mu \mathrm{mol} / \mathrm{L})$ & $606.9(92.4-1294.1)$ & $325.3(147.5-8 \mid 5.6)$ & I35.| (31.0-946.0) & $0.002^{\mathrm{a}}$ \\
\hline eGFR $\left(\mathrm{mL} / \mathrm{min} / \mathrm{I} .73 \mathrm{~m}^{2}\right)$ & $9.7(2.5-62.0)$ & $17.8(6.6-44.5)$ & $55.2(5.5-1 \mid 4.68)$ & $0.00 \mathrm{I}^{\mathrm{a}}$ \\
\hline \multicolumn{5}{|l|}{ 24-h urine output } \\
\hline Anuria & $2(8 \%)$ & 0 & 0 & 0.162 \\
\hline Oliguria & $8(32 \%)$ & $2(33 \%)$ & $4(20 \%)$ & 0.631 \\
\hline 24-h urine protein $(g / d)$ & $3.8(0-23.5)$ & $5.6 \mathrm{I}(2.2-9.1)$ & $5.2(0-18.7)$ & 0.428 \\
\hline Nephrotic-range proteinuria & $13(52 \%)$ & $3(50 \%)$ & $12(60 \%)$ & 0.924 \\
\hline Nephrotic syndrome & 0 & 0 & $8(40 \%)$ & $<0.00 I^{a, b}$ \\
\hline Albumin in urine protein (\%) & $23.0(1.0-70.5)$ & $17.0(2.3-3 \mid .6)$ & $73.6(4.6-85.5)$ & $0.012^{\mathrm{a}}$ \\
\hline \multicolumn{5}{|l|}{ Urine protein composition category } \\
\hline$<10 \%$ albumin & $6 / 15$ (40\%) & $2 / 3(67 \%)$ & $\mathrm{I} / \mathrm{I} 2(8 \%)$ & 0.054 \\
\hline $10 \%-50 \%$ & $3 / 15(20 \%)$ & $1 / 3(33 \%)$ & 0 & 0.181 \\
\hline$>50 \%$ albumin & $6 / 15(40 \%)$ & 0 & $11 / 12$ (92\%) & $0.002^{\mathrm{a}, \mathrm{b}}$ \\
\hline Microscopic hematuria & $3(12 \%)$ & 0 & $13(48 \%)$ & $<0.00 \mathrm{I}^{\mathrm{a}, \mathrm{b}}$ \\
\hline Requiring hemodialysis & $14(56 \%)$ & $3(50 \%)$ & $2(10 \%)$ & $0.004^{\mathrm{a}}$ \\
\hline \multicolumn{5}{|l|}{ Heavy chain type in serum } \\
\hline $\lg G$ & $2 / 24(8 \%)$ & $1 / 6(17 \%)$ & $7 / 20(35 \%)$ & 0.071 \\
\hline $\lg A$ & $2 / 24(8 \%)$ & 0 & $4 / 20(20 \%)$ & 0.46 \\
\hline $\lg M$ & 0 & 0 & $\mathrm{I} / 20(5 \%)$ & 0.52 \\
\hline None & $20 / 24(84 \%)$ & $5 / 6(83 \%)$ & $8 / 20(40 \%)$ & $<0.006^{\mathrm{a}}$ \\
\hline \multicolumn{5}{|l|}{ Light chain type in urine } \\
\hline$\kappa$ & $7 / 24(29 \%)$ & $3 / 6(50 \%)$ & $4 / 20(20 \%)$ & 0.41 \\
\hline$\lambda$ & I7/24 (7I\%) & $3 / 6(50 \%)$ & $12 / 20(60 \%)$ & 0.55 \\
\hline None & 0 & 0 & $4 / 20(20 \%)$ & 0.055 \\
\hline Bone lesions & $7(28 \%)$ & $3(50 \%)$ & $3(15 \%)$ & 0.208 \\
\hline Plasma cells on bone marrow smear (\%) & $33.5(10.0,79.5)$ & I7.0 (II.5, 84.5) & I5.8 $(10.0,32.5)$ & $0.013^{\mathrm{a}}$ \\
\hline
\end{tabular}

Notes: ${ }^{a}$ Pure $M C N$ vs non-MCN, $\mathrm{p}<0.05 ;{ }^{\mathrm{b}} \mathrm{MCN}+$ other pathologies vs non-MCN, $\mathrm{p}<0.05$.

Abbreviations: MCN, myeloma cast nephropathy; eGFR, estimated glomerular filtration rate; ESRD, end-stage renal disease.

number of myeloma casts (Table 6). The independent predictor of progression to ESRD in the multivariate analysis was the number of myeloma casts (HR: 1.08, 95\% CI: $1.08-1.15 ; \mathrm{p}=0.012$ ).
Of the 45 patients with available follow-up data, 17 (38\%) died, 6 of whom died within 12 months after kidney biopsy. The causes of death in our patients included myeloma (6 patients, 35\%), infection (4 patients, 23\%), 
Table 5 Treatment and Outcomes

\begin{tabular}{|c|c|c|c|c|}
\hline Characteristic & Pure MCN & MCN + Other Pathologies & Non-MCN & $\mathbf{P}$ \\
\hline Number of patients & 22 & 5 & 18 & \\
\hline Median duration of follow up, (months) & $14(3-9 \mid)$ & $50(2-58)$ & $25(2-109)$ & 0.187 \\
\hline Supportive therapy & 22 & 5 & 4 & $<0.001^{\mathrm{a}, \mathrm{b}}$ \\
\hline \multicolumn{5}{|l|}{ Chemotherapy } \\
\hline Proteasome inhibitors included & 17 (77\%) & $4(80 \%)$ & $10(56 \%)$ & 0.353 \\
\hline Other chemotherapy (VAD/MPT) & $3 / 2(23 \%)$ & $1 / 0(20 \%)$ & I/I (II\%) & 0.549 \\
\hline Proteasome inhibitors included + HDM/ASCT & 0 & 0 & $6(33 \%)$ & $0.006^{\mathrm{a}}$ \\
\hline \multicolumn{5}{|l|}{ Renal outcomes, number (\%) } \\
\hline Improved/stable & II (50\%) & $2(40 \%)$ & $13(72 \%)$ & 0.290 \\
\hline Worsening & 0 & I (20\%) & $3(17 \%)$ & 0.114 \\
\hline ESRD & II (50\%) & $2(40 \%)$ & $2(11 \%)$ & $0.021^{\mathrm{a}}$ \\
\hline Death, number (\%) & II (50\%) & I (20\%) & 5 (28\%) & 0.275 \\
\hline
\end{tabular}

Notes: ${ }^{a}$ Pure $\mathrm{MCN}$ vs non-MCN, $\mathrm{p}<0.05$; ${ }^{\mathrm{b}} \mathrm{MCN}+$ other pathologies vs non-MCN, $\mathrm{p}<0.05$.

Abbreviations: HDM/ASCT, autologous peripheral blood stem cell transplantation; VAD, vinorelbine, pirarubicin and dexamethasone; MPT, melphalan, prednisone and thalidomide; ESRD, end-stage renal disease.

cardiovascular diseases (3 patients, 18\%) and cerebrovascular disease (1 patient, $6 \%$ ). The cause of death was unknown in 3 patients $(18 \%)$. The median patient survival time was 54 months (95\% CI: 39.8-68.2), and the mean time from diagnosis to death was 28.2 months (range: 3-84) in patients who died.

No significant difference was found in the rate of death among the three groups. The median survival was 8.7 months in the pure MCN group and was significantly shorter than that in the non-MCN group (not reached); however, it was not significantly different from that in the $\mathrm{MCN}+$ other pathologies group (not reached; Figure 2B). Univariate analysis revealed that the predictors of patient death were male sex, the serum creatinine level, the degree of tubular atrophy, and the number of myeloma casts (Table 7). In the multivariate analysis, male sex (HR: 3.64; 95\% CI: 1.28-10.34; $\mathrm{p}=0.015$ ) and the number of myeloma casts (HR: 1.17 ; 95\% CI: $1.07-$ $1.29 ; \mathrm{p}=0.001$ ) were correlated with survival.

\section{Discussion}

In our study, pure MCN was the most common monoclonal immunoglobulin-associated lesion (49\%), followed by pure amyloidosis (17\%) and pure LCDD (10\%). The percentage of pure MCN in our cohort was higher than that in a previous report by Nasr et $\mathrm{al}^{7}$ (pure MCN, 28\%; pure amyloidosis, 18\%; pure MIDD, 17\%) and by Ivanyi et $\mathrm{l}^{15}$ (pure MCN, 32\%; pure amyloidosis, $11 \%$; pure LCDD, 5\%). However, more than two types of renal histopathologic patterns could be diagnosed with the same biopsy specimen. In our study, nearly $20 \%$ of patients with MCN had a second renal pathology pattern. Notably, 4 of $31 \mathrm{MCN}$ patients (13\%) had biopsy-proven
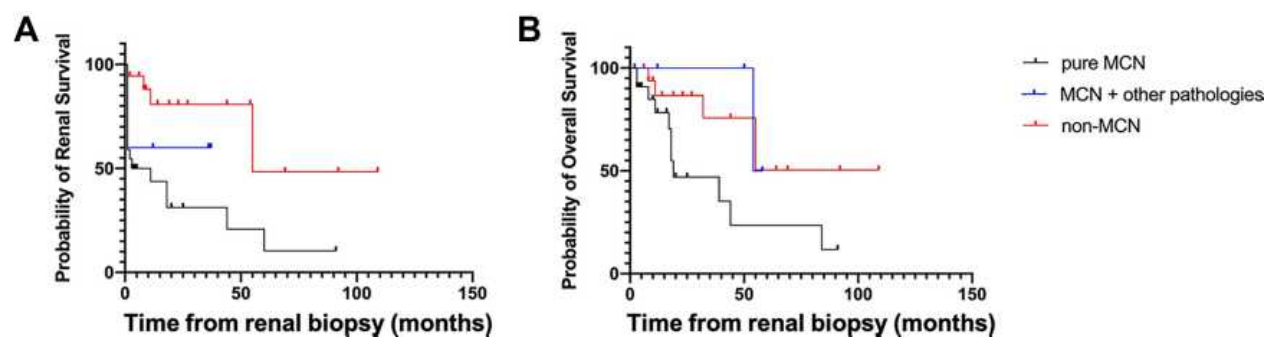

Figure 2 (A) shows renal survival of patients divided according to renal histology. The median renal survival was 6.9 months in the pure $M C N$ group and was not reached in the non-MCN and MCN + other pathologies groups. Log rank $p=0.0093$. (pure $M C N$ vs $M C N+$ other pathologies, $p=0.3993 ; p u r e ~ M C N$ vs non-MCN, $p=0.0021 ; M C N$ + other pathologies vs non- $\mathrm{MCN}, \mathrm{p}=0.2163$ ); (B) shows overall survival of patients divided according to renal histology. The median overall survival was 8.7 months in the pure $M C N$ group and was not reached in the non-MCN and $M C N+$ other pathologies groups. Log rank $p=0.0418$ (pure $M C N$ vs $M C N+$ other pathologies, $p=0.124$; pure $M C N$ vs non-MCN, $p=0.0298 ; M C N+$ other pathologies vs non- $M C N, p=0.800$ ). 
Table 6 Prognostic Factors Associated with Renal Survival

\begin{tabular}{|c|c|c|c|c|}
\hline \multirow[t]{2}{*}{ Parameters } & \multicolumn{2}{|l|}{ Univariate Analysis } & \multicolumn{2}{|c|}{ Multivariate Analysis } \\
\hline & HR (95\% CI) & $\mathbf{p}$ & HR (95\% Cl) & $\mathbf{p}$ \\
\hline Age & $0.98(0.95-1.02)$ & 0.37 & $0.997(0.96-1.04)$ & 0.890 \\
\hline Male & $2.13(0.92-4.94)$ & 0.78 & $1.81(0.79-4.1 \mathrm{I})$ & 0.159 \\
\hline Serum creatinine & $1.002(1.002-1.003)$ & $<0.001$ & - & - \\
\hline HDM/ASCT & $0.20(0.27-1.52)$ & 0.12 & - & - \\
\hline Number of myeloma casts & $1.09(1.03-1.15)$ & 0.002 & $1.08(1.08-1.15)$ & 0.012 \\
\hline Plasma cells on bone marrow smear (\%) & $1.01(0.99,1.03)$ & 0.062 & - & - \\
\hline Acute tubular injury & $2.27(0.96-5.40)$ & 0.063 & - & - \\
\hline Tubular atrophy & $1.60(0.94-2.7)$ & 0.082 & - & - \\
\hline Interstitial fibrosis & $1.08(0.61-1.93)$ & 0.79 & - & - \\
\hline Anemia & $1.91(0.83-4.42)$ & $0 .|3|$ & - & - \\
\hline
\end{tabular}

Note: Multivariate hazard ratio model: age, male gender and number of myeloma casts.

Abbreviation: HDM/ASCT, autologous peripheral blood stem cell transplantation.

Table 7 Prognostic Factors Associated with Overall Survival

\begin{tabular}{|c|c|c|c|c|}
\hline \multirow[t]{2}{*}{ Parameters } & \multicolumn{2}{|l|}{ Univariate Analysis } & \multicolumn{2}{|c|}{ Multivariate Analysis } \\
\hline & HR $(95 \% \mathrm{Cl})$ & $\mathbf{p}$ & HR (95\% Cl) & $\mathbf{p}$ \\
\hline Age & $0.75(0.29-1.98)$ & 0.6 & $1.02(0.96-1.08)$ & 0.479 \\
\hline Male & $2.89(1.07-7.76)$ & 0.04 & $3.64(1.28-10.34)$ & 0.015 \\
\hline Serum creatinine & $1.001(1.000-1.003)$ & 0.04 & - & - \\
\hline HDM/ASCT & $0.26(0.34-1.95)$ & 0.2 & - & - \\
\hline Number of myeloma casts & $1.09(1.02-1.16)$ & 0.02 & $1.17(1.07-1.29)$ & 0.001 \\
\hline Plasma cells on bone marrow smear (\%) & $1.02(0.99,1.04)$ & 0.084 & - & - \\
\hline Acute tubular injury & $1.78(0.67-4.75)$ & 0.248 & - & - \\
\hline Tubular atrophy & $3.5(1.19-10.27)$ & 0.023 & - & - \\
\hline Interstitial fibrosis & $1.35(0.75-2.43)$ & 0.311 & - & - \\
\hline Anemia & $1.009(0.50-2.05)$ & 0.981 & - & - \\
\hline
\end{tabular}

Note: Multivariate hazard ratio model: age, male gender and number of myeloma casts.

Abbreviation: HDM/ASCT, autologous peripheral blood stem cell transplantation.

renal LCDD. In a previous study by Mohan et al, 13 of 69 MM patients (13\%) with LCCD had biopsy-proven MCN. ${ }^{16}$ In another study reported by Zand et al, 13 of 42 MM patients (31\%) with MCN had biopsy-proven LCCD. ${ }^{9}$

We divided our cohort into 3 groups (pure MCN, MCN + other pathologies and non-MCN) and found many clinical differences. Patients with MCN were more likely to present with anemia, hypoproteinemia, and acute kidney disease and have isolated serum-free light chains than those with non-MCN. However, patients with non-MCN were more likely to present with nephrotic syndrome, albuminuria and microscopic hematuria. In summary, the clinical condition of renal damage in patients with pure MCN or MCN + other pathologies are more serious and more urgent.
Notably, according to our study, patients with pure MCN or MCN + other pathologies can also present with nephrotic-range proteinuria but not nephrotic syndrome. Two factors may explain this finding: (1) MCN patients presented with acute kidney injury that was found very early, thus, serum albumin did not have sufficient time to decrease to the hypoalbuminemia level; (2) proteinuria of most patients with MCN contains very little albumin, a possible reason why they did not present with nephrotic syndrome. Some studies have shown that renal impairment caused by MCN can be reversed and survival can be improved if an early diagnosis and treatment are available. ${ }^{17}$ These clinical differences may be helpful for distinguishing the type of pathology and determining logical treatment, particularly when patients are ineligible for a kidney biopsy. 
In our cohort, the median patient survival was 54 months, which was similar to that in previous studies $^{7,12,13,16}$ but longer than that in a much earlier report. ${ }^{18}$ The better patient survival over the past decade may be due to the introduction of new therapeutic regimens, including bortezomib, and improved supportive care and hemodialysis technology. The introduction of novel agents has improved renal impairment reversal, and favorable outcomes can be achieved in most patients with renal impairment when the appropriate therapy is promptly applied. ${ }^{19}$ Similar to previous study, myeloma, cardiovascular diseases and infection were the main causes of death in our study. ${ }^{20}$

Recent studies have demonstrated that, in MCN patients, the extent of cast formation, tubular atrophy and interstitial fibrosis were prognostic factors for the renal outcome. ${ }^{12,13}$ In our study, we also enrolled patients with non-MCN and suggested that the number of myeloma casts was an independent predictor of patient survival. Several factors can explain this finding: (1) MCN usually occurs in patients with a heavy tumor load (serum free light chain $>500 \mathrm{mg} / \mathrm{L}$ ), and more myeloma casts correspond to a heavier tumor load; ${ }^{17}$ (2) patients with MCN are more likely to progress to ESRD with maintenance dialysis, resulting in poor survival, ${ }^{20-22}$ and a large number of casts and diffuse tubular atrophy lead to a poor renal prognosis. ${ }^{12,13}$ For MM patients, dialysis is a risk factor for death. ${ }^{20}$

We also found that male sex was an independent predictor of overall survival. A previous study showed that, in MM patients, male sex was an independent risk factor for infection, ranking as the second most important cause of death in our cohort. ${ }^{23}$ Furthermore, a recent study showed that CKD in males progressed more quickly. ${ }^{24}$ The relationship between CKD and cardiovascular diseases is well established. The precise reason why male sex is an independent predictor requires further investigation.

The limitations of our study include the short follow-up time, unstandardized treatments, lack of other parameters, and the small sample size, which cannot represent the entire MM population. In our study, 132 patients (52\%) had a creatinine level greater than $178 \mu \mathrm{mol} / \mathrm{L}$, which is more than double that in a previous study in China. ${ }^{25}$ The reason may be a selection bias because our hospital has a highly regarded nephrology department, and patients with renal impairment are more likely to choose our hospital for treatment. Therefore, a large-sample, multicenter study with a long-term follow-up is needed.

\section{Conclusions}

In patients with newly diagnosed $\mathrm{MM}$, pure $\mathrm{MCN}$ is the most common monoclonal immunoglobulin-associated renal lesion. Patients with pure $\mathrm{MCN}$ or $\mathrm{MCN}+$ other pathologies had similar presentations, both having more severe renal injury and possibly presenting without nephrotic syndrome. The independent predictors of overall survival were male sex and the number of myeloma casts.

\section{Data Sharing Statement}

All data generated in this study are included in the main manuscript.

\section{Ethical Approval and Informed Consent}

All procedures performed in studies involving human participants were in accordance with the ethical standards of the Ethics Committee of the Peking University First Hospital (IRB approval number 2017[1280]) and with the 1964 Helsinki declaration and its later amendments or comparable ethical standards. A signed informed consent was obtained from all patients for participation in this study. All data used in this manuscript were anonymized.

\section{Funding}

This work was supported by the National Natural Science Foundation of China (Grant Number: 82070747), and CAMS Innovation Fund for Medical Sciences (2019-I2M -5-046). The funders had no role in study design, data collection, and analysis, interpretation of the data, the decision to publish, or preparation of the manuscript.

\section{Disclosure}

The authors declare that they have no conflicts of interests.

\section{References}

1. Rollig C, Knop S, Bornhauser M. Multiple myeloma. Lancet. 2015;385(9983):2197-2208. doi:10.1016/S0140-6736(14)60493-1

2. Eleutherakis-Papaiakovou V, Bamias A, Gika D, et al. Renal failure in multiple myeloma: incidence, correlations, and prognostic significance. Leuk Lymphoma. 2007;48(2):337-341. doi:10.1080/ 10428190601126602

3. Dimopoulos MA, Kastritis E, Rosinol L, Blade J, Ludwig H. Pathogenesis and treatment of renal failure in multiple myeloma. Leukemia. 2008;22(8):1485-1493. doi:10.1038/leu.2008.131

4. Motwani SS, Herlitz L, Monga D, Jhaveri KD, Lam AQ. Paraproteinrelated kidney disease: glomerular diseases associated with paraproteinemias. Clin j Am Society Nephrol. 2016;11 (12):2260-2272. doi:10.2215/CJN.02980316 
5. Lin ZS, Qin AB, Wang SX, et al. A non-invasive differential diagnostic model for light chain cast nephropathy in newly diagnosed multiple myeloma patients with renal involvement: a multicenter study. J Nephrol. 2021. doi:10.1007/s40620-020-00926-7

6. Sethi S, Rajkumar SV, D'Agati VD. The complexity and heterogeneity of monoclonal immunoglobulin-associated renal diseases. J Am Society Nephrol. 2018;29(7):1810-1823. doi:10.1681/ASN.2017121319

7. Nasr SH, Valeri AM, Sethi S, et al. Clinicopathologic correlations in multiple myeloma: a case series of 190 patients with kidney biopsies. Am j Kidney Dis. 2012;59(6):786-794. doi:10.1053/j.ajkd.2011.12.028

8. Nasr SH, Valeri AM, Cornell LD, et al. Renal monoclonal immunoglobulin deposition disease: a report of 64 patients from a single institution. Clin $j$ Am Society Nephrol. 2012;7(2):231-239. doi:10.2215/CJN.08640811

9. Zand L, Nasr SH, Gertz MA, et al. Clinical and prognostic differences among patients with light chain deposition disease, myeloma cast nephropathy and both. Leuk Lymphoma. 2015;56 (12):3357-3364. doi:10.3109/10428194.2015.1040011

10. Lin J, Markowitz GS, Valeri AM, et al. Renal monoclonal immunoglobulin deposition disease: the disease spectrum. J Am Society Nephrol. 2001;12(7):1482-1492. doi:10.1681/ASN.V1271482

11. Dimopoulos MA, Sonneveld P, Leung N, et al. International myeloma working group recommendations for the diagnosis and management of myeloma-related renal impairment. J clin oncol. 2016;34 (13):1544-1557. doi:10.1200/JCO.2015.65.0044

12. Ecotiere L, Thierry A, Debiais-Delpech C, et al. Prognostic value of kidney biopsy in myeloma cast nephropathy: a retrospective study of 70 patients. Nephrol Dial Transplant. 2016;31(1):64-72. doi:10.1093/ndt/gfv283

13. Royal V, Leung N, Troyanov S, et al. Clinicopathologic predictors of renal outcomes in light chain cast nephropathy: a multicenter retrospective study. Blood. 2020;135(21):1833-1846. doi:10.1182/ blood.2019003807

14. Durie BG, Kyle RA, Belch A, et al. Myeloma management guidelines: a consensus report from the Scientific Advisors of the International Myeloma Foundation. Hematol J. 2003;4(6):379-398. doi:10.1038/sj.thj.6200312

15. Iványi B. Frequency of light chain deposition nephropathy relative to renal amyloidosis and Bence Jones cast nephropathy in a necropsy study of patients with myeloma. Arch Pathol Lab Med. 1990;114 (9):986-987.
16. Mohan M, Buros A, Mathur P, et al. Clinical characteristics and prognostic factors in multiple myeloma patients with light chain deposition disease. Am J Hematol. 2017;92(8):739-745. doi:10.1002/ajh.24756

17. Hutchison CA, Cockwell P, Stringer S, et al. Early reduction of serum-free light chains associates with renal recovery in myeloma kidney. J Am Society Nephrol. 2011;22(6):1129-1136. doi:10.1681/ ASN.2010080857

18. Montseny JJ, Kleinknecht D, Meyrier A, et al. Long-term outcome according to renal histological lesions in 118 patients with monoclonal gammopathies. Nephrol Dial Transplant. 1998;13(6):1438-1445. doi:10.1093/ndt/13.6.1438

19. Gavriatopoulou M, Terpos E, Kastritis E, Dimopoulos MA. Current treatments for renal failure due to multiple myeloma. Expert Opin Pharmacother. 2016;17(16):2165-2177. doi:10.1080/ 14656566.2016.1236915

20. Decourt A, Gondouin B, Delaroziere JC, et al. Trends in survival and renal recovery in patients with multiple myeloma or light-chain amyloidosis on chronic dialysis. Clin $j$ Am Society Nephrol. 2016;11(3):431-441. doi:10.2215/CJN.06290615

21. Dimopoulos MA, Roussou M, Gavriatopoulou M, et al. Outcomes of newly diagnosed myeloma patients requiring dialysis: renal recovery, importance of rapid response and survival benefit. Blood Cancer J. 2017;7(6):e571. doi:10.1038/bcj.2017.49

22. Cobb J, Plantinga L, Luthi JC, et al. Pre-ESRD care and mortality in incident ESRD patients with multiple myeloma. Am J Clin Oncol. 2018;41(4):367-370. doi:10.1097/COC.0000000000000275

23. Sørrig R, Klausen TW, Salomo M, Vangsted A, Gimsing P. Risk factors for infections in newly diagnosed Multiple Myeloma patients: a Danish retrospective nationwide cohort study. Eur J Haematol. 2019;102(2):182-190. doi:10.1111/ejh.13190

24. Ricardo AC, Yang W, Sha D, et al. Sex-related disparities in CKD Progression. $J$ Am Society Nephrol. 2019;30(1):137-146. doi:10.1681/ASN.2018030296

25. Lu J, Lu J, Chen W, et al. Clinical features and treatment outcome in newly diagnosed Chinese patients with multiple myeloma: results of a multicenter analysis. Blood Cancer J. 2014;4:e239. doi:10.1038/ bcj. 2014.55

\section{Publish your work in this journal}

Cancer Management and Research is an international, peer-reviewed open access journal focusing on cancer research and the optimal use of preventative and integrated treatment interventions to achieve improved outcomes, enhanced survival and quality of life for the cancer patient.
The manuscript management system is completely online and includes a very quick and fair peer-review system, which is all easy to use. Visit http://www.dovepress.com/testimonials.php to read real quotes from published authors. 